\title{
Two loop mass effects in the static position space QCD-potential
}

\author{
Michael Melles ${ }^{\mathrm{a}}$ \\ ${ }^{\text {aP Particle Theory }}$ \\ Paul Scherrer Institute \\ Villigen, CH-5232, Switzerland
}

\begin{abstract}
The perturbatively calculable short distance QCD potential is known to two loops including the effect of massive quarks. Recently, a simple approximate solution in momentum space was utilized to obtain the potential in coordinate space. The latter is important in several respects. A comparison with non-perturbative lattice results is feasible in the overlap regime using light $\overline{\mathrm{MS}}$ masses. This might be even more promising employing the concept of the force between the heavy color singlet sources, which can be easily derived from the potential. In addition, the better than two percent accuracy bottom mass determination from $\Upsilon$-mesons is sensitive to massive charm loops at the two loop order. We summarize recent results using exact one loop functions and explicit decoupling parametrizations.
\end{abstract}

In analogy to QED, the heavy quark potential [1.2] is of central interest in QCD as a measure of the strong coupling. In the long distance regime $\sim \mathcal{O}(1 \mathrm{fm})$ it contains the confining linear tail. With the inclusion of dynamical quarks on the lattice, one expects to enter the so called "string breaking" regime at distances of $\mathcal{O}(2 \mathrm{fm})$ yielding the famous Yukawa potential 33. At very short distances $(\leq 0.1 \mathrm{fm})$ one recovers the Coulombpart of the potential, modified by loop corrections like in QED. The overlap region is naturally of considerable interest since non-perturbative effects might also enter [4] and possibly as a "normalization" for lattice calculations. The important point to notice here is that fermions with light $\overline{\mathrm{MS}}$-masses are easier to implement on the lattice. For a direct comparison, due to its smaller value, the force [5] between the static sources is also of special interest. For a recent review of lattice results on the forces in heavy bound states see Ref. [6]. From a phenomenological point of view, the importance of calculating mass effects in the perturbative part of the heavy quark potential is two fold. On the one hand, since we are dealing with a physical system, the corresponding physical coupling definition (V-scheme) has several welcome advantages compared to massless (calculational) schemes. $\alpha_{V}\left(Q^{2} / m^{2}\right)$ is an observable i.e. the flavor thresholds are analytic, display au- tomatic decoupling of heavy quarks and are independent of the renormalization scale to the order we are working. In addition, the physical scale of the problem is determined by the transfered momentum. Detailed discussions of the flavor threshold treatment using analyitc schemes are contained in Refs. [7 [9]. On the other hand, the two loop mass corrections to the heavy quark potential are important for the better than two percent accuracy determination of the bottom mass [10]. In this case it is important not to treat the charm loops as massless but to consider the full massive calculation. Using the physical $\Upsilon$-meson for this purpose, the effect of the mass shift $\delta m_{b}$ depends on $\left\langle\phi_{1 s}\left|V_{F}\left(r, m_{c}\right)\right| \phi_{1 s}\right\rangle$, where $\phi_{1 s}=\frac{1}{\sqrt{\pi}}\left(\frac{m_{b} C_{F} \alpha_{s}}{2}\right)^{\frac{3}{2}} \exp \left(-\frac{C_{F} \alpha_{s}}{2} m_{b} r\right)$ denotes the 1s ground state wave function of the $\Upsilon$-meson and $V_{F}$ the massive fermionic corrections to the potential. Note that in momentum space one would have an additional integration since each wave function depends on a different three momentum making the overall calculation prohibitive.

We begin, by recalling the definition of the potential through the manifestly gauge invariant vacuum expectation value of the Wilson loop $W_{\Gamma}=\left\langle 0\left|\operatorname{Tr} \mathcal{P} \exp \left(i g \oint_{\Gamma} d x_{\mu} A_{a}^{\mu} \mathrm{T}^{a}\right)\right| 0\right\rangle$ of spatial extension $r$ and large temporal extension $T$ with gluon exchanges between the temporal lines. The 
path-ordering $\mathcal{P}$ is necessary due to the noncommutativity of the $\mathrm{SU}(3)$ generators $\mathrm{T}^{a}$. In the perturbative analysis through two loops considered here, all spatial components of the gauge fields $A_{a}^{\mu}(\mathbf{r}, \pm T / 2)$ can at most depend on a power of $\log T$ and are thus negligible here. Furthermore, $W_{\Gamma} \stackrel{T \rightarrow \infty}{\longrightarrow} \exp \left(-i T E_{0}\right)$, where the ground state energy $E_{0}$ is identified with the potential $V$. Thus we arrive at the definition:

$$
\begin{aligned}
& V(r, m)=-\lim _{T \rightarrow \infty} \frac{1}{i T} \\
& \log \left\langle 0\left|\operatorname{Tr} \mathcal{P} \exp \left(i g \oint_{\Gamma} d x_{\mu} A_{a}^{\mu} \mathrm{T}^{a}\right)\right| 0\right\rangle
\end{aligned}
$$

Writing the source term of the heavy charges, separated at the distance $r \equiv\left|\mathbf{r}-\mathbf{r}^{\prime}\right|$, as

$J_{\mu}^{a}(x)=i g v_{\mu} T^{a}\left[\delta(\mathbf{x}-\mathbf{r})-\delta\left(\mathbf{x}-\mathbf{r}^{\prime}\right)\right]$

and neglecting contributions connecting the spatial components, the perturbative potential is given by

$$
\begin{aligned}
& V(r, m)=-\lim _{T \rightarrow \infty} \frac{1}{i T} \\
& \log \left\langle 0\left|\operatorname{Tr} \mathcal{T} \exp \left(\int d^{4} x A_{\mu}^{a}(x) J_{a}^{\mu}(x)\right)\right| 0\right\rangle
\end{aligned}
$$

In the above equation $v_{\mu}=\delta_{\mu, 0}$ due to the purely timelike nature of the sources. The potential in momentum space is the Fourier transform of $V(r)$. It can be calculated directly in momentum space from the on-shell heavy quark anti-quark scattering amplitude (divided by i) at the physical momentum transfer $\mathbf{q}$, projected onto the color singlet sector. The potential can be used to define the effective charge $\alpha_{V}$ (the so-called V-scheme) through:

$$
\begin{aligned}
V(Q, m) & \equiv-4 \pi C_{F} \frac{\alpha_{V}(Q, m)}{Q^{2}} \\
V(r, m) & \equiv-C_{F} \frac{\alpha_{V}(r, m)}{r}
\end{aligned}
$$

where $Q^{2} \equiv \mathbf{q}^{2}=-q^{2}$ and both expressions above are related through a Fourier-transform, i.e. $\quad V(r, m)=\int \frac{d^{3} Q}{(2 \pi)^{3}} V(Q, m) \exp (i \mathbf{Q r}) . \quad$ At lowest order we obtain therefore the well known Coulomb potential $V_{C}$ in each representation.
In Ref. [11] the two loop corrections in momentum space were given in approximate form based on the reconstructed solutions of the GellMann Low function with massive quarks obtained in Ref. [9] from the exact numerical results in Ref. [12]. In order to render decoupling of heavy flavors with pole mass $m$ explicit one has to use the decoupling relation [13]:

$$
\begin{aligned}
& \alpha_{s}^{\left(n_{l}\right)}(\mu)=\alpha_{s}^{\left(n_{l}-1\right)}(\mu)\left\{1+\frac{\alpha_{s}^{\left(n_{l}-1\right)}(\mu)}{\pi} \frac{1}{6} \log \frac{\mu^{2}}{m^{2}}+\right. \\
& \left.\left(\frac{\alpha_{s}^{\left(n_{l}-1\right)}(\mu)}{\pi}\right)^{2}\left[\frac{7}{24}+\frac{19}{24} \log \frac{\mu^{2}}{m^{2}}+\frac{1}{36} \log ^{2} \frac{\mu^{2}}{m^{2}}\right]\right\}(6)
\end{aligned}
$$

It is then useful to write the mass corrections to the potential in such a way that the light quark with mass $m$ is included in the evolution of the strong coupling. Thus the mass corrections vanish in the limits $m \longrightarrow 0$ and $Q^{2} \longrightarrow \infty$. We find from the results of Ref. 11] the following expression:

$V^{\mathrm{NNL}}(Q, m)=V^{\mathrm{NNL}}(Q, 0)+\delta V^{\mathrm{NNL}}(Q, m)$

where the first term on the r.h.s. is given in Ref. (14) correcting an error in the original calculation of the pure glue part of Ref. [15,16]. Denoting the one loop subtracted mass correction function by

$\mathrm{P}\left(\frac{m^{2}}{Q^{2}}\right) \equiv \frac{5}{3}-\log \frac{Q^{2}}{m^{2}}+2 Q^{2} \int_{1}^{\infty} \frac{d x f(x)}{Q^{2}+4 m^{2} x^{2}}$

with

$f(x) \equiv \sqrt{x^{2}-1} \frac{2 x^{2}+1}{2 x^{4}}$

we find

$\delta V^{\mathrm{NNL}}(Q, m)=V_{C}\left(Q^{2}\right)\left\{\frac{\alpha_{\mathrm{MS}}^{\left(n_{l}\right)}(\mu)}{4 \pi} \frac{4}{3} T_{F} \mathrm{P}\left(\frac{m^{2}}{Q^{2}}\right)\right.$

$\left(\frac{\alpha \frac{\left(n_{l}\right)}{\mathrm{MS}}(\mu)}{4 \pi}\right)^{2}\left[\frac{8}{3} T_{F} \mathrm{P}\left(\frac{m^{2}}{Q^{2}}\right)\left(\frac{31}{9} C_{A}-\frac{20}{9} T_{F} n_{l}\right.\right.$

$\left.-\beta_{0} \log \frac{Q^{2}}{\mu^{2}}\right)+\frac{16}{9} T_{F}^{2} \mathrm{P}^{2}\left(\frac{m^{2}}{Q^{2}}\right)+\frac{76}{3} T_{F} \times$

$\left[c_{1} \int_{c_{2}}^{\infty} \frac{d x}{x} \frac{2 Q^{2}}{Q^{2}+4 m^{2} x^{2}}+d_{1} \int_{d_{2}}^{\infty} \frac{d x}{x} \frac{2 Q^{2}}{Q^{2}+4 m^{2} x^{2}}\right.$

$\left.\left.+\frac{161}{114}+\frac{26}{19} \zeta_{3}-\log \frac{Q^{2}}{m^{2}}\right]\right\}$ 
The fitting constants are given by $c_{2}=0.47 \pm 0.01$, $d_{2}=1.12 \pm 0.02$ with $c_{1}, d_{1}$ fixed by the two conditions $c_{1}+d_{1}=1$ and $c_{1} \log \left(4 c_{2}^{2}\right)+d_{1} \log \log \left(4 d_{2}^{2}\right)=$ $\frac{161}{114}+\frac{26}{19} \zeta_{3}$ in order to ensure exact decoupling when using relation 6. Eq. 10 can be used to obtain the corresponding results in coordinate space. Here we find analogously (with $\hat{m}=e^{\gamma} m$ ):

$$
\begin{aligned}
& \delta V^{\mathrm{NNL}}(r, m)=V_{C}(r)\left\{\frac { \alpha _ { \overline { \mathrm { MS } } } ^ { ( n _ { l } ) } ( \mu ) } { 3 \pi } \left[\log (\hat{m} r)+\frac{5}{6}+\right.\right. \\
& \left.\int_{1}^{\infty} d x e^{-2 m r x} f(x)\right]+\left(\frac{\alpha_{\mathrm{MS}}^{\left(n_{l}\right)}(\mu)}{3 \pi}\right)^{2}\left[-\frac{3}{2} \int_{1}^{\infty} d x f(x)\right. \\
& e^{-2 m r x}\left(\beta_{0}\left(\log \frac{4 m^{2} x^{2}}{\mu^{2}}-\operatorname{Ei}(2 m r x)-\operatorname{Ei}(-2 m r x)\right)\right. \\
& \left.-\frac{31}{9} C_{A}+\frac{20}{9} T_{F} n_{l}\right)+\beta_{0} \frac{\pi^{2}}{4}+3\left(\log (\hat{m} r)+\frac{5}{6}\right) \times \\
& \left(\beta_{0} \log (\hat{\mu} r)+\frac{31}{18} C_{A}-\frac{10}{9} T_{F} n_{l}\right)-\int_{1}^{\infty} d x f(x) e^{-2 m r x} \\
& \left(\frac{1}{x^{2}}+x f(x) \log \frac{x-\sqrt{x^{2}-1}}{x+\sqrt{x^{2}-1}}-\operatorname{Ei}(2 m x r)-\right. \\
& \left.\operatorname{Ei}(-2 m x r)+\log \left(4 x^{2}\right)\right)+\frac{\pi^{2}}{12}+\left(\log (\hat{m} r)+\frac{5}{6}\right)^{2} \\
& +\frac{57}{4}\left(\frac{161}{228}+\frac{13}{19} \zeta_{3}+\log (\hat{m} r)+c_{1} \operatorname{Ei}\left(1,2 c_{2} m r\right)\right. \\
& \left.\left.\left.+d_{1} \operatorname{Ei}\left(1,2 d_{2} m r\right)\right)\right]\right\}
\end{aligned}
$$

where $\operatorname{Ei}(1, x)=\int_{x}^{\infty} \exp (-t) \frac{d t}{t}$. The relation $\operatorname{Ei}(x)+\operatorname{Ei}(-x)=\mathrm{P} \int_{0}^{\infty} \exp [(1-t) x] \frac{2 t d t}{1-t^{2}}$ is also useful, with $\mathrm{P}$ denoting the principal value prescription. While Eq. 11 vanishes for $m \longrightarrow 0$, it does not for $r \longrightarrow 0$. It is a rather interesting fact that through the Fourier transform of Eq. 10 a non-analytic "hinear mass term is generated which is furthermore enhanced by a factor $\pi^{2}$. It is important to note that the linear mass term cannot be obtained by first expanding Eq. 11 and then integrating over the dispersion relation variable $x$, since it originates from large values of $x$. This means it originates from momenta smaller than

\footnotetext{
${ }^{1}$ Non-analytic refers here to the fact that Eq. 12 should be read as being proportional to $\sqrt{m^{2}}$ since $m^{2}$ is the only mass term entering the momentum space result in Eq. 10 .
}

the quark mass, i.e. it is of infrared origin. We find the following limit:

$$
\begin{aligned}
& \delta V^{\mathrm{NNL}}(r, m) \stackrel{r \ll 1 / m}{\longrightarrow}-m \frac{C_{F}}{4}\left(\alpha_{\left.\frac{\left(n_{l}\right)}{\mathrm{MS}}(\mu)\right)^{2}\{1+}\right. \\
& \frac{\alpha_{\frac{\left(n_{l}\right)}{\mathrm{MS}}(\mu)}^{2 \pi}\left[\frac{31}{9} C_{A}-\frac{20}{9} T_{F} n_{l}+\beta_{0}\left(\log \frac{\mu^{2}}{m^{2}}-4 \log (2)\right.\right.}{\left.\left.+3)+\frac{4}{45}(31-30 \log (2))+\frac{76}{3 \pi}\left(c_{1} c_{2}+d_{1} d_{2}\right)\right]\right\}} \\
& +\mathcal{O}\left(m^{2} r\right)
\end{aligned}
$$

In terms of the $\overline{\mathrm{MS}}$-mass parameter $\bar{m}(\mu)$, one only needs to use the relation

$m=\bar{m}(\mu)\left[1+C_{F} \frac{\alpha_{\overline{\mathrm{MS}}}(\mu)}{\pi}\left(1+\frac{3}{2} \log \frac{\mu}{\bar{m}(\mu)}\right)\right]$

in the one loop term to obtain the NNL correction. The size of the mass corrections relative to the Born Coulomb potential is displayed in Fig. 1 for the charm quark mass $m=1.5 \mathrm{GeV}$ and the "natural" renormalization scale choice $\mu=\frac{1}{r e^{\gamma}}$, since the $\gamma$ terms are generated by the Fourier transform. In Ref. [17] it is shown that this scale choice is almost identical to the BLM-scale 18 and thus consistently absorbs large renormalization group logarithms. The two loop running coupling is given by

$$
\alpha_{\overline{\mathrm{MS}}}(\mu)=\frac{4 \pi}{\beta_{0} \log \frac{\mu^{2}}{\Lambda_{Q C D}^{2}}}\left(1-\frac{\beta_{1}}{\beta_{0}^{2}} \frac{\log \left(\log \frac{\mu^{2}}{\Lambda_{Q C D}^{2}}\right)}{\log \frac{\mu^{2}}{\Lambda_{Q C D}^{2}}}\right)
$$

where we normalize the QCD-scale parameter $\Lambda_{Q C D}$ such that $\alpha_{\overline{\mathrm{MS}}}\left(M_{Z}\right)=0.12$ which corresponds to $\Lambda_{Q C D}=0.25 \mathrm{GeV}$ and we keep $n_{l}=4$ fixed. The first two terms of the $\beta$-function are gauge invariant and scheme independent in massless renormalization schemes and are given by $\beta_{0}=\frac{11}{3} C_{A}-\frac{4}{3} T_{F} n_{l}$ and $\beta_{1}=\frac{34}{3} C_{A}^{2}-\frac{20}{3} C_{A} T_{F} n_{l}-$ $4 C_{F} T_{F} n_{l}$. In QCD we have $C_{A}=3, C_{F}=\frac{4}{3}$ and $T_{F}=\frac{1}{2}$. The effect is at the several percent level and increases above $10 \%$ for distances of $0.1 \mathrm{fm}$ $\left(\sim 0.5 \mathrm{GeV}^{-1}\right)$. It vanishes for small $r$ since we are displaying the corrections relative to $V_{C}$. In absolute terms we checked numerically that Eq. 12 is reproduced for small distances by the full 


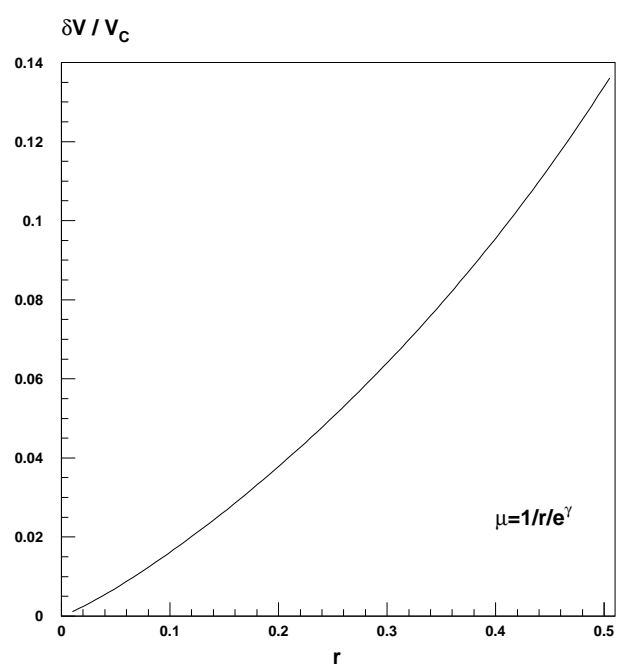

Figure 1. The size of the charm-mass corrections in Eq. 11 relative to the Coulomb potential. The choice of the natural renormalization scale is indicated in the figure. The distance $r$ between the sources is in $\mathrm{GeV}^{-1}$.

result in Eq. 11. The inclusion of these charm quark corrections into a full sum rule determination of the bottom quark mass in Ref. [19] led to a shift of $-30 \mathrm{MeV}$ and yields $\bar{m}_{b}\left(\bar{m}_{b}\right)=4.17 \pm 0.05$ $\mathrm{GeV}$, which in light of the small error is a significant contribution.

Instead of considering the potential, or $\alpha_{V}(r, m)$, it is also of interest to study the coupling $\alpha_{F}(r, m)=-r^{2} \frac{\partial\left(\alpha_{V}(r, m) / r\right)}{\partial r}$ which is defined from the force between the static quarks. In general $\alpha_{F}(r, m)$ is smaller than $\alpha_{V}(r, m)$ or even $\alpha_{\overline{\mathrm{MS}}}(\mu=1 / r)$ [11], which makes it suitable as an expansion parameter in perturbative calculations. It is also useful in lattice calculations to determine $\alpha_{s}$ 20,21]. The force is given by $F(r, m)=-\frac{\partial V(r, m)}{\partial r}$ and the massless case is taken from Ref. [11]:

$F^{\mathrm{NNL}}(r, 0)=-C_{F} \frac{\alpha_{\overline{\mathrm{MS}}}^{\left(n_{l}\right)}(\mu)}{r^{2}}\left\{1+\frac{\alpha_{\overline{\mathrm{MS}}}^{\left(n_{l}\right)}(\mu)}{4 \pi}(\right.$
$\left.2 \beta_{0} \log (\hat{\mu} r)+f_{1}\right)+\left(\frac{\alpha_{\frac{\left(n_{l}\right)}{\mathrm{MS}}(\mu)}}{4 \pi}\right)^{2}\left(4 \beta_{0}^{2} \log ^{2}(\hat{\mu} r)\right.$ $\left.\left.+2\left[\beta_{1}+2 \beta_{0} f_{1}\right] \log (\hat{\mu} r)+f_{2}\right)\right\}$

where

$$
\begin{aligned}
& f_{1}=-\frac{35}{9} C_{A}+\frac{4}{9} T_{F} n_{l} \\
& f_{2}=\left(-\frac{7513}{162}+\frac{229}{27} \pi^{2}-\frac{1}{4} \pi^{4}+\frac{22}{3} \zeta_{3}\right) C_{A}^{2}+ \\
& \left(\frac{3410}{81}-\frac{88}{27} \pi^{2}-\frac{56}{3} \zeta_{3}\right) C_{A} T_{F} n_{l}-\left(\frac{31}{3}-\right. \\
& \left.16 \zeta_{3}\right) C_{F} T_{F} n_{l}-\left(\frac{560}{81}-\frac{16}{27} \pi^{2}\right)\left(T_{F} n_{l}\right)^{2}
\end{aligned}
$$

From Eq. 11 we thus find for the mass corrections the following expression:

$$
\begin{aligned}
& \delta F^{\mathrm{NNL}}(r, m)=-\frac{\partial \log V_{C}(r)}{\partial r} \delta V^{\mathrm{NNL}}(r, m)-V_{C}\{ \\
& \frac{\alpha_{\overline{\mathrm{MS}}}^{\left(n_{l}\right)}(\mu)}{3 \pi}\left[\frac{1}{r}-\int_{1}^{\infty} d x 2 m x f(x) e^{-2 m r x}\right]+\left(\frac{\alpha_{\overline{\mathrm{MS}}\left(n_{l}\right)}(\mu)}{3 \pi}\right)^{2} \\
& \times\left[\frac { 3 } { 2 } \int _ { 1 } ^ { \infty } d x e ^ { - 2 m r x } f ( x ) \left(2 m x \left(\beta _ { 0 } \left[\log \frac{4 m^{2} x^{2}}{\mu^{2}}-\right.\right.\right.\right. \\
& \left.\operatorname{Ei}(2 m r x)-\operatorname{Ei}(-2 m r x)]-\frac{31}{9} C_{A}+\frac{20}{9} T_{F} n_{l}\right)+ \\
& \left.\frac{\beta_{0}}{r}\left(e^{2 m r x}+e^{-2 m r x}\right)\right)+\frac{3}{r} \beta_{0}\left[\log (\hat{m} r)+\frac{5}{6}\right] \\
& +\frac{3}{r}\left(\beta_{0} \log (\hat{\mu} r)+\frac{31}{18} C_{A}-\frac{10}{9} T_{F} n_{l}\right)+ \\
& \int_{1}^{\infty} d x e^{-2 m r x} f(x)\left(2 m x \left(\log \left(4 x^{2}\right)-\operatorname{Ei}(2 m r x)-\right.\right. \\
& \left.\operatorname{Ei}(-2 m r x)+\frac{1}{x^{2}}+x f(x) \log \frac{x-\sqrt{x^{2}-1}}{x+\sqrt{x^{2}-1}}\right)+ \\
& \left.\frac{1}{r}\left(e^{2 m r x}+e^{-2 m r x}\right)\right)+\frac{2}{r}\left(\log (\hat{m} r)+\frac{5}{6}\right)+ \\
& \left.\left.\frac{57}{4 r}\left(1-c_{1} e^{-2 c_{2} m r}-d_{1} e^{-2 d_{2} m r}\right)\right]\right\}
\end{aligned}
$$

The size of the charm-mass corrections relative to the Born term is presented in Fig. 2 for the same scale choice as in Fig. I. It can be seen that 


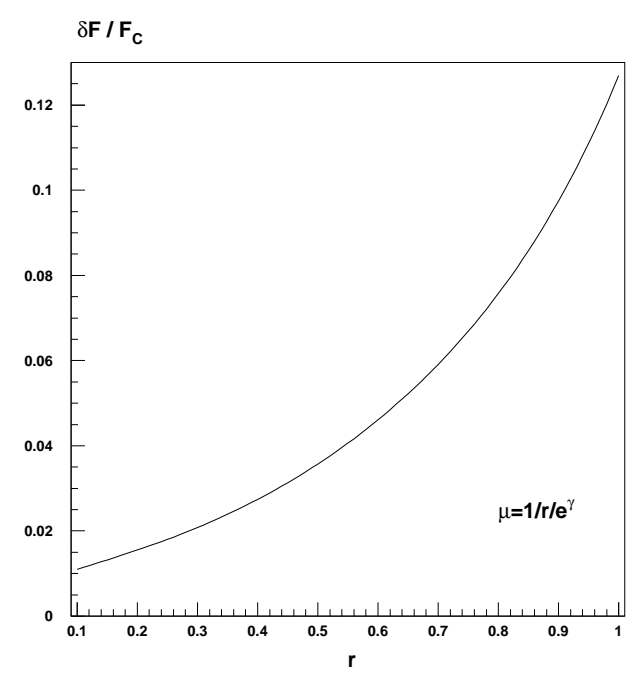

Figure 2. The size of the charm-mass corrections in Eq. 18 relative to the lowest order force from the Coulomb potential. The choice of the natural renormalization scale is indicated in the figure. The distance $r$ between the sources is in $\mathrm{GeV}^{-1}$.

the effect is less than half of that for the mass corrections to the potential and start to increase more rapidly at distances over $0.1 \mathrm{fm}$.

In summary, we have calculated the massive quark corrections to the static QCD potential in coordinate space at the two loop level. The results presented here use the exact one loop vacuum polarization functions and a dispersion relation fit based on the results of Ref. [11]. The uncertainty is estimated at the percentile level from comparisons with the exact Monte Carlo results in momentum space of Ref. [12]. For the bottom mass determination, the inclusion of a massive charm quark in the $\Upsilon$ potential is significant due to two reasons. One factor is that the effective physical scale depends parametrically on the charm mass, leading to a large value of the coupling. The other reason originates from the fact that in the relation between the potential contribution to the static energy and the pole mass, there is an uncanceled non-analytic linear mass term [10.19], whose origin is the Fourier transform. Together, these two effects lead to a shift of $-30 \mathrm{MeV}$ and $\bar{m}_{b}\left(\bar{m}_{b}\right)=4.17 \pm 0.05 \mathrm{GeV}$. In addition we have calculated the mass corrections for the force between two static color charges in a singlet state at the two loop level. The size of the effect in general is smaller than for the potential but still significant at larger distances. At small distances, the linear $r$-independent mass term from the potential drops out and thus leads to smaller corrections in this regime.

\section{REFERENCES}

1. L. Susskind, lectures given at Les Houches 1976, North Holland 1977, 207.

2. W. Fischler, Nucl. Phys. B129, 157, 1977.

3. H. Yukawa, Proc. Phys. Math. Soc. Jap.17:48, 1935.

4. G.S. Bali, Phys. Lett. B460:170, 1999.

5. G. Grunberg, Phys. Rev. D40, 680, 1989.

6. G.S. Bali, hep-ph/0001312.

7. S.J. Brodsky, M.S. Gill, M. Melles, J. Rathsman. Phys. Rev. D58:116006, 1998.

8. M. Melles, Nucl. Phys. Proc. Suppl.86:211, 2000 and ICHEP 98, Vancouver 1998, high energy physics, vol. 1; 718.

9. S.J. Brodsky, M. Melles, J. Rathsman, Phys. Rev. D60:096006, 1999.

10. A.H. Hoang, A.V. Manohar, Phys. Lett. B483:94, 2000.

11. M. Melles, hep-ph/0001295, to appear in Phys. Rev. D.

12. M. Melles, Phys. Rev. D58:114004, 1998.

13. S.A. Larin, T.van Ritbergen, J.A. Vermaseren, Nucl. Phys. B438, 278, 1995.

14. Y.Schröder, Phys. Lett. B447:321, 1999 and Nucl. Phys. Proc. Suppl. 86:525, 2000.

15. M. Peter, Phys. Rev. Lett. 78:602, 1997; Nucl. Phys. B501:471, 1997.

16. M. Jezabek, M. Peter, Y. Sumino, Phys. Lett. B428:352, 1998.

17. M. Peter, Doctoral Thesis, Karlsruhe University, 1997.

18. S.J. Brodsky, G.P. Lepage, P.B. Mackenzie, Phys. Rev. D28:228, 1983.

19. A.H. Hoang, hep-ph/0008102.

20. C. Michael, Phys. Lett. B283:103, 1992.

21. R. Sommer, Nucl. Phys. B411:839, 1994. 